Synaptic Plasticity-Molecular, Cellular, and Functional Aspects. Edited by MICHEL BAUDRY, RICHARD F THOMPSON and JOEL L DAVIS. Published by The MIT Press, London 1994. (Pp 258; £44.95). ISBN 0-262-02359-8.

The ability of synapses to change their properties, either by gain or loss of entire synapses or by alteration of efficiency of signal transduction within individual synapses, is an essential feature of complex nervous systems that alter their response over time. While learning and memory represent perhaps the most obvious application of this "plasticity", the changes seen during development and in association with degenerative disease are also very striking. The clinical significance of synaptic plasticity is obvious; degenerative disorders of memory represent a massive workload for neurologists and an extremely expensive drain on health care resources. A book exarnining the molecular and cellular biology of the mechanisms of plasticity is therefore a welcome and potentially valuable addition if it is able to bring together the advances in the many different areas under study within this field so as to provide a coherent framework that both provides insight into basic mechanisms and also goes on to suggest therapeutic approaches.

This book represents a series of reviews written by individual laboratories. The emphasis is firmly on the molecular mechanisms responsible for alteration in synaptic efficiency, with the bulk of the book concerned with the important phenomena of long term potentiation (LTP) and long term depression (LT.D). In these phenomena, synaptic transmission is potentiated or depressed by prior experience to tetanic stimuli. Such changes in synaptic efficiency could obviously provide a mechanism of altering the pathway responses based on previous experience and so provide a synaptic pathway capable of "learning". The elucidation of the channels involved and the central role of calcium and NMDA receptors has been a tremendous advance over the past few years and the work has provided new approaches of memory disorders. This work is well described and clearly referenced, with additional chapters on the functional implications for those who wish to pursue the subject in further depth.

Outside the areas of LTP and LTD, there are two chapters in this book examining other areas of plasticity that I enjoyed particularly. Firstly, Dr Rose provides a lucid account of learning in a valuable model system, the newborn chick. While many will be concerned that the complexity of this system will make it difficult to dissect the molecular mechanisms of learning, there is no doubt that the chick provides an excellent example of learning in a higher vertebrate and one that should be of great value to those exploring therapeutic approaches to learning disorders. Secondly, a chapter by Dr Steward covers another aspect of plasticity; the evidence for local synthesis of synaptic protein. Given that any neuron has many dendrites, all of which have synapses from different inputs and all of which can potentially be altered by experience, it follows that the cell has the difficulty of regulating many different synapses individually. There is growing evidence that, at least in part, this is performed by local synthesis of synaptic protein within the dendrites. This is quite differen from the more usual mechanism of protein synthesis around the nucleus and subsequent translocation to the site of insertion. A method using local synthesis allows greater flexibility as each area could be individually controlled. The evidence for this has emerged largely from Dr Steward's laboratory in Charlottesville, Virginia and is described very clearly in his chapter. It is important work that may provide some of the answers as to how individual neurons can be so flexible in their response.

There are, however, a number of other areas of synaptic plasticity that are covered in rather less detail. Development is associated with considerable synaptic change, as shown very clearly by the in vivo studies of single mouse neuromuscular junctions, over many weeks, in living animals by Lichmann and colleagues at Washington University, St Louis. The mechanism by which this change occurs is very important, and I was disappointed to see so little attention paid to development in this book. There is only one short chapter, which concentrates on the role of the extracellular matrix and the mechanisms of stabilisation. I would have enjoyed a more detailed review of the evidence for plasticity as a key developmental process. Another area of research that has added to our understanding of learning and memory is studies in the sea slug, Aplysia, by Kandel and colleagues at Columbia University, New York showing a fascinating range of electrical and structural changes associated with learning. This work illustrates very clearly the value of studying simple model systems, and a review would have been a valuable contribution to this book.

Overall, then, I enjoyed the book and found the chapters well written and informative. I was, however, disappointed by the omissions. I do not think this book is appropriate for general readership in light of its rather focused approach. It will provide a valuable source of reference for students of learning and memory and would be a valuable addition to specific libraries, but it is not a book to buy for your bookshelf at home.

\section{CHARLES FFRENCH-CONSTANT}

Neuropsychology-A Clinical Approach. Third Edition. By KEVIN waLSH. Published by Churchill Livingstone, Edinburgh 1994. (Pp 465; £29.95). ISBN 443049378.

The appearance of this now classic text in the 1970s helped establish neuropsychology as a field of neuroscience. Since then, the subject has grown rapidly. The development of behavioural neurology and neuropsychiatry creates a need for neurologists and psychiatrists to become acquainted with neuropsychology.

The main branch of neuropsychology nowadays is cognitive. Theoretical models are constructed which aim to explain normal mental processes, while elucidation of the neuroanatomical structures involved is given somewhat less prominence. Walsh, however, adopts a "psychoanatomical" approach, that is, the study of brain damaged patients primarily to understand the neuro-anatomical localisation of psychological function; this is felt to be more useful in helping the clinician understand individual cases. The concept of the "distributed anatomical system" is described, in which each psychological function utilises several interconnected anatomical sites. Similarly, individual cortical areas are involved in several psychological functions. Although this muddies the waters of localisation of function, it is a more realistic representation of cognitive function.

In line with this anatomical stance, each lobe has a separate chapter. This leads to distributed cognitive functions, such as memory, being described in several chapters. An alternative division on the basis of cognitive functions, for example memory, language, would make cognition less intimidating to the beginner. As the psychoanatomical approach has been largely superseded by cognitive neuropsychology, the references are somewhat dated.

The book will prove useful to psychologists as an introduction to the field, although they may be more comfortable with the cognitive approach. By contrast, clinical neurologists, given their neuroanatomical training, may find the cognitive method initially somewhat alien to the medical mind, and may find the brain-behaviour approach a more useful introductory approach to neuropsychology.

JOHN GREENE

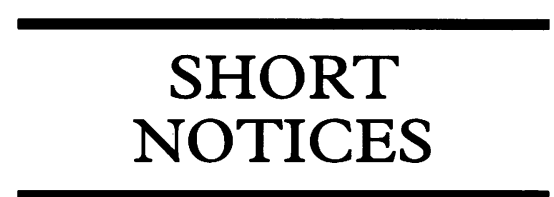

Readers may be interested in: Alcohol and Alcohol Problems. Edited by G EDWARDS and T J PETERS. (Pp 240 illustrated; $£ 49.95$.) Published by Churchill Livingstone, Edinburgh 1994. ISBN 443051518.

Biomagnetic Stimulation. Edited by SHOOGO UENO. (Pp 136; \$65.) Published by Plenum Press, New York 1994. ISBN 0-306-44707-X.

Neutral Activity and the Growth of the Brain. By DALE PURVES. (Pp 108; $£ 24.95$ $\mathrm{H} / \mathrm{b}, £ 11.95 \mathrm{P} / \mathrm{b}$.) Published by Cambridge University Press, Cambridge 1994. ISBN 0-521-45496-4 H/b 0-521-45570-7 P/b. 